\title{
High flow nasal cannula in acute hypercapnic exacerbation of chronic obstructive pulmonary disease: an emerging utility
}

\author{
Anshul Mittal', Megha Varshney ${ }^{2}$, Vidushi Rathi ${ }^{1}$, Pranav Ish ${ }^{1}$ \\ ${ }^{1}$ Department of Pulmonary, Critical Care And Sleep Medicine, Vardhman Mahavir Medical College and Safdarjung \\ Hospital, New Delhi; ${ }^{2}$ Department of Medicine, Vardhman Mahavir Medical College And Safdarjung Hospital, New \\ Delhi, India
}

\begin{abstract}
High flow nasal cannula (HFNC) provides warmed and humidified air with flow rates up to 60 liters/min with relatively fixed oxygen content $\left(\mathrm{FiO}_{2}\right)$. It has been extensively evaluated for hypoxemic respiratory failure and has been used in mild acute respiratory distress syndrome, pre-intubation, bronchoscopy and pediatric obstructive sleep apnea. Recent data has suggested a role in stable hypercapnic chronic obstructive pulmonary disease (COPD) and even in acute exacerbations, though, the use has not been advocated by any guidelines yet. We present a case of acute hypercapnic exacerbation of COPD, intolerant to non-invasive ventilation, showing response and improvement on use of HFNC. This case highlights this potential mechanisms and prospects for the same.
\end{abstract}

Correspondence: Pranav Ish, Department of Pulmonary, Critical Care and Sleep Medicine, Vardhman Mahavir Medical College and Safdarjung Hospital, New Delhi, India. Tel. +91.9958356000. E-mail: pranavish2512@gmail.com

Contributions: All the Authors contributed equally. All the Authors read and approved the final version of the manuscript and agree to be accountable for all aspects of the work.

Conflict of interest: The Authors declare no conflict of interest.

Informed consent: Written informed consent was obtained from a legally authorized representative(s) for anonymized patient information to be published in this article.

Key words: High flow; exacerbation; hypercapnia.

Received for publication: 24 August 2019.

Accepted for publication: 15 December 2019.

${ }^{\circ}$ Copyright: the Author(s), 2020

Licensee PAGEPress, Italy

Monaldi Archives for Chest Disease 2020; 90:1158

doi: 10.4081/monaldi.2020.1158

This article is distributed under the terms of the Creative Commons Attribution Noncommercial License (by-nc 4.0) which permits any noncommercial use, distribution, and reproduction in any medium, provided the original author(s) and source are credited.

\section{Introduction}

Although non-invasive positive-pressure ventilation (NIV) is the therapy of choice for acute hypercapnic respiratory failure in chronic obstructive pulmonary disease (COPD), some patients are intolerant to its use. High flow nasal cannula (HFNC) supportive therapy has emerged as a safe, useful therapy in patients with respiratory failure, improving oxygenation and comfort. We hereby report a case of hypercapnic respiratory failure with intolerance for use of NIV where HFNC use was successful.

\section{Case Report}

A 75-year lady, current smoker and diagnosed COPD, on irregular inhaled dual bronchodilators for 10 years, was admitted with complaints of progressively worsening dyspnoea following fever for two days and increased cough with expectoration, both in amount and purulence. The patient had no co-morbidities like diabetes, hypertension or coronary artery disease.

On general examination, the patient was conscious but agitated, with a pulse rate of $90 / \mathrm{min}$, respiratory rate of $28 / \mathrm{min}$ and blood pressure of $118 / 82 \mathrm{mmHg}$. On systemic examination, bilateral rhonchi were heard in lung fields, with rest of examination being within normal limits. Presenting arterial blood gas revealed respiratory acidosis as summarized in Table 1 along with other laboratory parameters. The patient had neutrophilic leukocytosis with preserved organ functions.

On echocardiography, her right atrium and ventricle were dilated and left ventricle ejection fraction (LVEF) was 65\% with pulmonary artery pressures of $30 \mathrm{mmHg}$. Chest $\mathrm{x}$-Ray was suggestive of bilateral emphysematous lung field with flattening of diaphragm (Figure 1). On lung ultrasound no B lines or $\mathrm{C}$ pattern was appreciated and there was no evidence of effusion. Patient was uncooperative for use of NIV with oro-nasal mask and consent for invasive mechanical ventilation was denied by kin.

Hence, high flow oxygen through nasal cannula at a flow rate of $30-35 \mathrm{~L} / \mathrm{min}$ and $\mathrm{FiO}_{2}$ of around $30 \%$ (to target saturation of 88 $92 \%$ ) was used along with parenteral antibiotic, oral steroids and nebulization with bronchodilators (levalbuterol and ipratropium) (Figures 2 and 3). The patient's vital and consciousness status were monitored at regular interval of one hour and arterial blood gas was repeated at 4,8 and $12 \mathrm{~h}$ (Table 1). Patient clinical condition improved gradually, and her arterial blood gas normalized after $12 \mathrm{~h}$ with resolution of respiratory acidosis with patient becoming alert and oriented. The respiratory rate also decreased 
from 28 to 18/min. She was discharged on day 6 post admission with advice of home oxygen and inhaled bronchodilators. The leukocytosis had resolved as well as fever. She was asked to follow up after 2 weeks for further therapy optimization, rehabilitation, and plan for long term oxygen therapy.

\section{Discussion}

HFNC has many uses (Table 2). The above described case presents as an uncommon occasion were HFNC was used to manage a case of acute hypercapnic respiratory failure in a COPD successfully. In patient with acute hypercapnic respiratory failure, NIV has been the primary modality to improve tidal volume and maintain alveolar ventilation hence improving oxygenation and resolving hypercapnia. But around $25 \%$ of patients have contraindication for use of NIV and another $15 \%$ tolerate it poorly due to mask intolerance, claustrophobia and development of

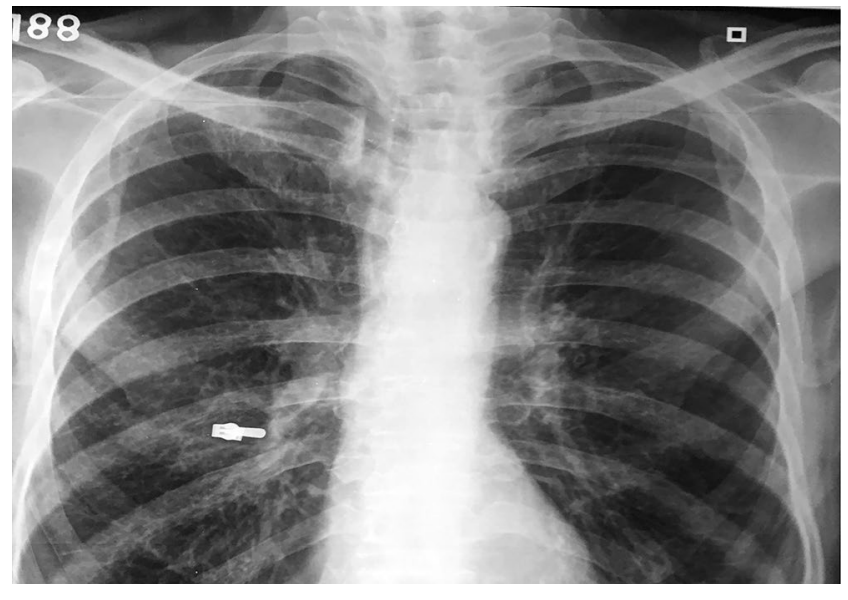

Figure 1. Chest $x$-ray showing hyperinflation with no evidence of consolidation.

Table 1. Laboratory parameters and serial ABG values.

\begin{tabular}{|c|c|c|c|c|}
\hline Parameter & $\begin{array}{l}0 \text { hour } \\
\text { (room air) }\end{array}$ & $\begin{array}{c}4^{\text {th }} \text { hour } \\
\left(\mathrm{FiO}_{2} 30 \%-\right. \\
\text { flow rate } 35 \mathrm{~V} / \mathrm{min})\end{array}$ & $\begin{array}{l}12^{\text {th }} \text { hour } \\
\left(\mathrm{FiO}_{2} 30 \%-\right. \\
\text { flow rate } 35 \mathrm{~V} / \mathrm{min})\end{array}$ & $\begin{array}{l}\text { Pre-discharge } \\
\text { (room air) }\end{array}$ \\
\hline $\mathrm{pH}$ & 7.23 & 7.33 & 7.39 & 7.41 \\
\hline $\mathrm{PO}_{2}(\mathrm{mmHg})$ & 38 & 56 & 58 & 46 \\
\hline $\mathrm{PCO}_{2}(\mathrm{mmHg})$ & 64 & 50 & 46 & 50 \\
\hline $\mathrm{HCO}_{3}$ & 28 & 27 & 27 & 30 \\
\hline $\mathrm{SO}_{2}(\%)$ & 65 & 88 & 90 & 83 \\
\hline $\mathrm{Hb}($ gram\%) & 14.6 & & & 14.5 \\
\hline TLC & 16000 & & & 9000 \\
\hline Neutrophil (\%) & 90 & & & 70 \\
\hline Platelet count & 400,000 & & & 360,000 \\
\hline Blood urea (mg/dl) & 20 & & & 21 \\
\hline Serum creatinine & 0.7 & & & 0.8 \\
\hline Total bilirubin & 1.1 & & & 1.0 \\
\hline AST/ALT & $34 / 35$ & & & $38 / 39$ \\
\hline ALP & 51 & & & 46 \\
\hline
\end{tabular}

$\mathrm{ABG}$, arterial blood gas analysis; $\mathrm{PO}_{2}$, partial pressure of oxygen; $\mathrm{PCO}_{2}$, partial pressure of carbon dioxide; $\mathrm{SO}_{2}$, oxygen saturation; $\mathrm{HB}$, hemoglobin; $\mathrm{TLC}$, total leukocyte count; $\mathrm{AST}$, aspartate transaminase; $\mathrm{ALT}$, alanine transaminase; ALP, alkaline phosphatase.

Table 2. Current indications of using HFNC (adapted from references 4-8).

\section{Indications}

1 Acute cardiogenic pulmonary edema

2 Mild to moderate ARDS

3 Pre, during and post intervention like Bronchoscopy

4 Pre-intubation

5 Do-not-intubate status in terminally ill patients

6 Immunocompromised patients with respiratory failure

7 OSA, especially in children

HFNC, high flow nasal cannula; ARDS, acute respiratory distress syndrome; OSA, obstructive sleep apnea.

pressure sore or dryness of throat [1]. In such patients HFNC can

be used as an alternate mode of ventilation to prevent intubation 
and provide them ventilator support. The device uses an oxygen air blender with an actively heated humidifying device which can provide $\mathrm{FiO}_{2}$ from 21 to $60 \%$ at a flow rate of as high as 60 $1 / \min [2]$.

HFNC cannot actively enhance tidal volume, it mainly functions in hypercapnic failure by flushing the pharyngeal dead space and improving alveolar ventilation. HFNC also improves thoracoabdominal synchrony, thereby decreasing respiratory rate. Hence, eventually the minute ventilation falls without reducing alveolar ventilation and $\mathrm{PCO}_{2}$ is reduced. Another suggested mechanism is that although delivered through open system, high flow overcomes resistance against expiratory flow and creates positive nasopharyngeal pressure which helps to recruit lung and decrease the work of breathing [3].

The used of humidified air increases muco-ciliary function to provide effective clearing of airway and associated with less atelectasis which improves ventilation perfusion matching providing better oxygenation at a lower $\mathrm{FiO}_{2}$. These are particularly important in patients of COPD which have secretions and are at risk of oxygen toxicity at higher $\mathrm{FiO}_{2}$ [4]. All these mechanisms have been summarized in Table 3. Evidence in hypoxemic respiratory failure is robust but no official guidelines exist for the same yet [5-7]. There are studies showing a potential benefit of HFNC in stable COPD but use in acute COPD is recent [9,10]. A recent study of over 50 stable hypercapnic COPD also indicated a favorable response in terms of rapid shallow breathing index (RSBI) and even $\mathrm{PCO}_{2}$ levels. This implored for further studies in acute hypercapnic COPD patients [10]. The GOLD 2019 guidelines also mention regarding the role of HFNC in COPD in hypoxemic respiratory failure. They have no mention for use of HFNC in hypercapnic respiratory failure due to acute exacerbation.

An observational retrospective cohort study in acute hypercapnic COPD patients presenting to emergency care comparing NIV and HFNC found comparable failure rates with evidence of decreased skin breakdown complications and nursing care requirements in HFNC patients. In fact, the duration of HFNC use was superior to NIV with a high tolerance rates, and most common cause of NIV failure was intolerance. However, again, the study had a retrospective cohort with a small sample size and itself warrants need for well-designed randomized control trials [11]. A single randomized trial in China has demonstrated that HFNC decreases diaphragmatic fatigue in acute exacerbation of COPD without much difference in arterial blood analysis [12]. Another randomized control trial of over 200 patients comparing HFNC and NIV in undifferentiated emergency setting respiratory failure also conclusively showed that HFNC is non-inferior. The subgroup of patients which had hypercapnia at presentation (30\%) also had similar ventilatory improvements in PCO2 levels. Patients could easily communicate and feed, decreasing anxiety levels, thereby increasing the practical utility [13].

Hence, to conclude HFNC can be used as an alternative mode of ventilation in COPD patients presenting as acute exacerbation with contraindication or intolerance to use of NIV. But still further research needs to be done regarding timing the start, monitoring and end point of therapy for patient in whom its use is warranted.

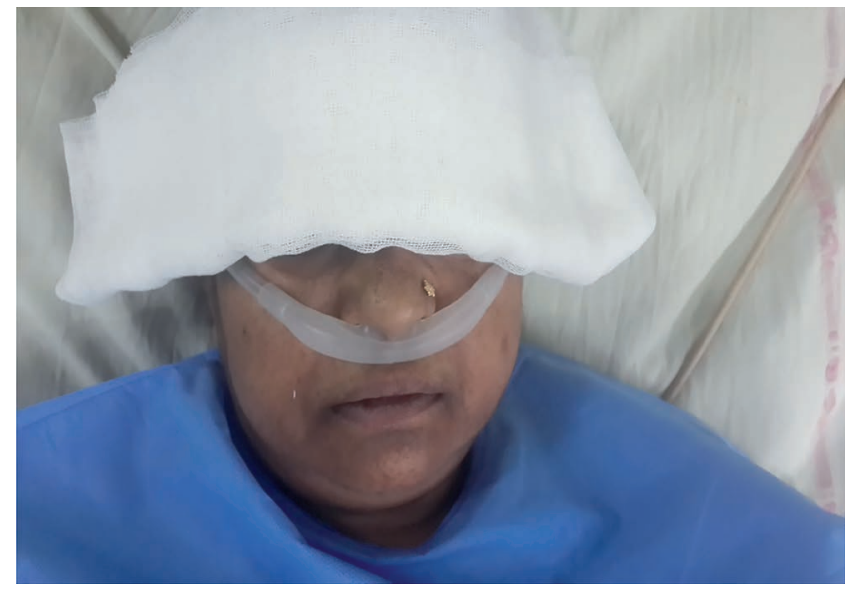

Figure 2. Thick nasal cannula of HFNC snugly fit in nostrils giving humidified high flow.

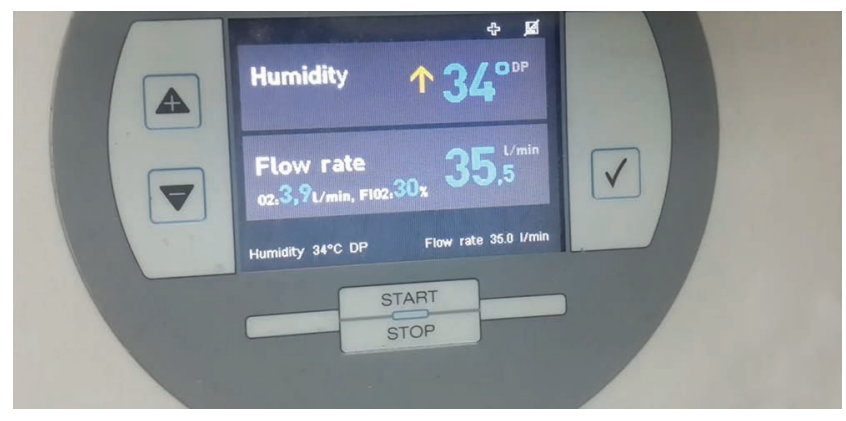

Figure 3. Screen showing $35 \mathrm{1} / \mathrm{min}$ flow with $\mathrm{FiO}_{2}$ of $30 \%$ being delivered by HFNC.

Table 3. Mechanisms responsible for HFNC success in acute hypercapnic respiratory failure of COPD (adapted from references 10-13).

\section{Mechanism}

\section{High flow washing out dead space}

2 Improved thoraco-abdominal synchrony improving alveolar ventilation

3 No claustrophobia

4 High flow creating a small PEEP effect and recruitment

5 Humidified and moist air to improve comfort and manage secretions

6 Fixed FiO2 preventing oxygen toxicity

HFNC, high flow nasal cannula; $\mathrm{COPD}$, chronic obstructive pulmonary disease; $\mathrm{PEEP}$, positive end-expiratory pressure; $\mathrm{FiO}_{2}$, fraction of inspired oxygen. 


\section{References}

1. Liu J, Duan J, Bai L. Noninvasive ventilation intolerance: characteristics, predictors, and outcomes. Respir Care 2016;61:277-84.

2. Itagaki T, Okuda N, Tsunano Y, et al. Effect of high-flow nasal cannula on thoraco-abdominal synchrony in adult critically ill patients. Respir Care 2014;59:70-4.

3. Lampland AL, Plumm B, Meyers PA, et al. Observational study of humidified highflow nasal cannula compared with nasal continuous positive airway pressure. J Pediatr 2009;154:177-82.

4. Saslow JG, Aghai ZH, Nakhla TA, et al. Work of breathing using high-flow nasal cannula in preterm infants. J Perinatol 2006;26:476-80.

5. Frat JP, Thille AW, Mercat A, et al. High-flow oxygen through nasal cannula in acute hypoxemic respiratory failure. N Engl J Med 2015;372:2185-96.

6. Roca O, Riera J, Torres F, et al. High-flow oxygen therapy in acute respiratory failure. Respir Care 2010;55:408-13.

7. Lenglet H, Sztrymf B, Leroy C et al. Humidified high flow nasal oxygen during respiratory failure in the emergency depart $\neg$ ment: feasibility and efficacy. Respir Care 2012;57: 1873-8.
8. Sztrymf B, Messika J, Bertrand F, et al. Beneficial effects of humidi $\neg$ fied high flow nasal oxygen in critical care patients: a prospective pilot study. Intens Care Med 2011;37:1780-6.

9. Pisani L, Fasano L, Corcione N, et al. Change in pulmonary mechanics and the effect on breathing pattern of high flow oxygen therapy in stable hypercapnic COPD. Thorax 2017;72:373-5.

10. Bräunlich J, Köhler M, Wirtz H. Nasal highflow improves ventilation in patients with COPD. Int J Chron Obstruct Pulmon Dis 2016;11:1077-85.

11. Sun J, Li Y, Ling B, et al. High flow nasal cannula oxygen therapy versus non-invasive ventilation for chronic obstructive pulmonary disease with acute-moderate hypercapnic respiratory failure: an observational cohort study. Int J Chron Obstruct Pulmon Dis 2019;14:1229-37.

12. Yang S, Zhang G, Liu Z, et al. [Effect of high-flow nasal cannula oxygen therapy on diaphragmatic function in patients with acute exacerbation of chronic obstructive pulmonary disease: a prospective randomized controlled trial].[Article in Chinese]. Zhonghua Wei Zhong Bing Ji Jiu Yi Xue 2019;31:551-5.

13. Doshi P, Whittle JS, Bublewicz M, et al. High-Velocity nasal insufflation in the treatment of respiratory failure: A randomized clinical trial. Ann Emerg Med 2018;72:73-83. 